\title{
3D Magnetic Field Modeling of a New Superconducting Synchronous Machine Using Reluctance Network Method
}

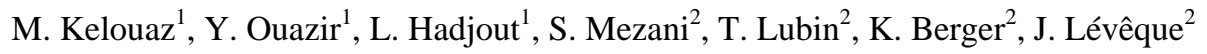 \\ ${ }^{1}$ LSEI-Université des Sciences et Technologie Houari Boumediene, BP Nº32, 16111, Alger, Algérie \\ ${ }^{2}$ GREEN-Université de Lorraine, Faculté des Sciences et Technologies, BP 239, 54506, Vandœuvre-lès-Nancy, France
}

\begin{abstract}
In this paper a new superconducting inductor topology intended for synchronous machine is presented. The studied machine has a standard 3-phase armature and a new kind of 2-poles inductor (claw-pole structure) excited by two coaxial superconducting coils. The air-gap spatial variation of the radial flux density is obtained by inserting a superconducting bulk which deviates the magnetic field due to the coils. The complex geometry of this inductor usually needs 3D finite elements (FEM) for its analysis. However, to avoid a long computational time inherent to 3D FEM, we propose in this work an alternative modeling which uses a 3D meshed reluctance network. The results obtained with the developed model are compared to 3D FEM computations as well as to measurements carried out on a laboratory prototype. Finally, a 3D FEM study of the shielding properties of the superconducting screen demonstrates the suitability of using a diamagnetic-like model of the superconducting screen.
\end{abstract}

Index Terms - Synchronous machine, inductor, 3D reluctances network, superconducting coils and bulks

\section{INTRODUCTION}

$\mathrm{T}$ HE use of superconducting materials (SC) in electrical machines allows to consider new structures and topologies of inductors to achieve new synchronous machines [1], [2]. With the technical progresses achieved in the manufacture of these materials, it is now possible to produce excitation coils supporting strong currents, bulk SC magnets with high residual flux density or SC screens that can expel strong magnetic field. By combining these last possibilities, several inductor structures of synchronous machines can be considered. The principle is based on the modulation of the magnetic field obtained by the exciting coils using superconducting screens [3]-[5]. High air-gap flux density values are achieved which result in compact machines suitable in marine, wind turbine and aircraft industries [2], [3]-[6]. The design of such superconducting machines requires the study of highly non-linear magnetic field diffusion in superconducting materials (excitation coils and screen).

The machine studied in this paper has been developed and constructed in the GREEN laboratory [4]. As shown in Fig. 1, it consists of a rotating conventional stator and a fixed superconducting inductor. The inductor is made with two coaxial superconducting coils separated by an inclined superconducting bulk. Thanks to the screening effect of the bulk, the axial field generated by the two coils is reoriented radially to obtain an electrical machine with one pole-pair.

The geometry of the studied inductor is complex and needs a 3D model for its analysis. The 3D finite elements method is a powerful tool but leads to very high computation time. We propose to model the considered structure with a 3D reluctance network method.

The reluctance network method has been already used for modeling reluctance machines, induction machines and permanent magnet machines [7], [8], [9]. Two approaches are commonly used:

The first approach is based on the decomposition of the structure into a set of flux tubes modeled by reluctances. The flux lines are then a priori known which reduces the flexibility of the model.
The second approach is a mesh-based one in which the studied domain is subdivided into small elements each of which is represented by reluctances.

This second approach consists on meshing the studied structure with surface elements in $2 \mathrm{D}$ and volume elements in $3 \mathrm{D}$. The flux flows in all directions, so the magnetic field line trajectories are not imposed [10].

This technique can also incorporate the movement between the rotor and the stator [11].

To the authors' knowledge, the use of this method for superconducting devices is extremely rare or non-existent. Compared with 3D FEM, this method can lead to a compromise between computation time and accuracy. In this paper, we present the 3D model formulation of the studied superconducting inductor using the second approach of the reluctance network method. The difficulty of adapting this method to the studied inductor structure is in the determination of a suitable mesh size to represent the superconductor screen placed between the two coils as shown in Fig. 1 .

The results obtained with the proposed magnetostatic method are compared with those issued form 3D finite elements simulations and experimental ones.

Finally, a 3D FEM study of the shielding properties of the superconducting bulk is conducted. A highly non-linear magnetic field diffusion problem is solved to demonstrate the suitability of using a diamagnetic-like model for the superconducting bulk.

\section{DESCRIPTION OF THE STUDIED SUPERCONDUCTING INDUCTOR}

The operation of the studied superconducting inductor is based on the principle of field screening using SC bulks. It is composed of two coaxial SC coils supplied by a dc current and separated by an inclined elliptical shape SC bulk (Fig.1.a). The axial magnetic field created by the two solenoids is diverted by the SC bulk which results in two magnetic poles mainly oriented in the radial direction. To increase the magnetic field created by this inductor and reduce the leakage flux, ferromagnetic parts can be inserted between the coils and 
the screen (Fig.1.b). By adding an external three-phase armature, we obtain a 2-pole synchronous machine (Fig.1.c).

This machine can be seen as a claw pole type synchronous machines which can develop a high electromagnetic torque due to the increase in the level of flux density in the air gap [6].

This is the structure, with complex geometry, that we propose to model with the meshed reluctance network approach.

For the validation of the model, a laboratory prototype for which we have some measured data is used. The two coils are wound with low critical temperature wire $(\mathrm{NbTi})$ and the SC bulk is made from YBCO material. Iron parts are added as shown in Fig.1.b. The obtained inductor is cooled with liquid helium at $4.2 \mathrm{~K}$.

The main geometric parameters of the studied inductor are shown in Fig.2 and their values given in Table I.

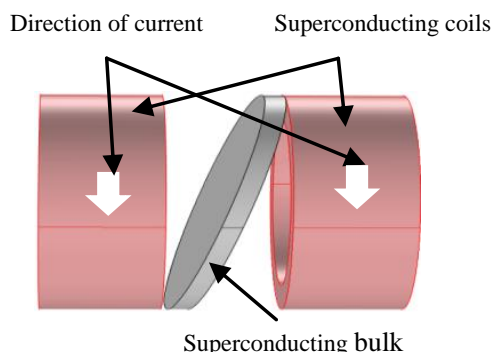

(a)- Without iron

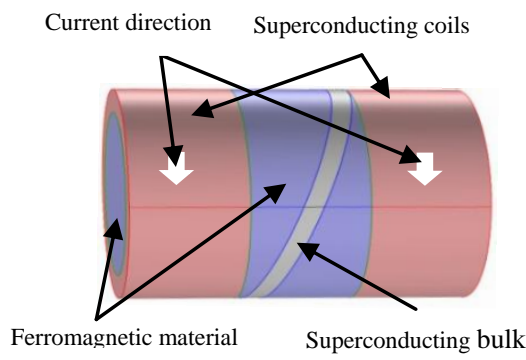

(b)- With iron



(c)- With iron and armature

Fig.1. Structure of the studied machine

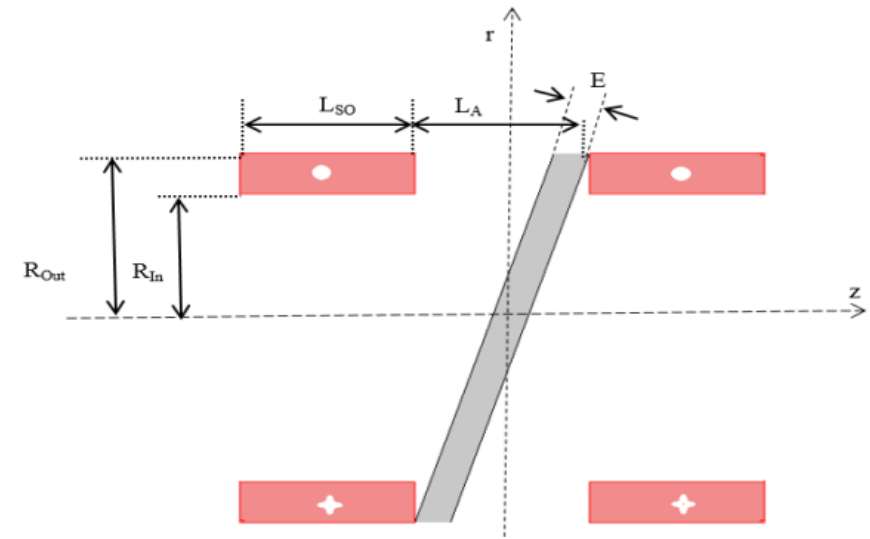

Fig.2. Inductor geometrical parameters

TABLE I

MAIN PARAMETERS OF THE STUDIED DEVICE

\begin{tabular}{cccc}
\hline \hline Symbol & Designation & Value & Units \\
\hline \hline NbTi & Solenoid wire material & - & - \\
$d c$ & NbTi round wire diameter & 0.7 & $\mathrm{~mm}$ \\
$R_{\text {Out }}$ & Solenoids outer radius & 68 & $\mathrm{~mm}$ \\
$R_{I n t}$ & Solenoids inner radius & 53 & $\mathrm{~mm}$ \\
$L_{A}$ & Distance between solenoids & 45 & $\mathrm{~mm}$ \\
$L_{S O}$ & Length of the solenoid & 45 & $\mathrm{~mm}$ \\
YBCO & SC bulk material & - & - \\
$E$ & Thickness of the SC bulk & 10 & $\mathrm{~mm}$ \\
\hline \hline
\end{tabular}

\section{Reluctance Network Model}

A meshed reluctance network method is used here to model, in 3D, the studied SC inductor.

This method consists of meshing the geometry by dividing it into a set of volume elements with a given geometric form. As for the finite element method, each generated volume element represents a material node characterized by:

- a constant permeability

- reluctances in the different directions

- $\mathrm{mmfs}$ if the volume element is located in the region of field source

The different nodes are then connected together through their reluctances in all directions. The application of Kirchhoff's laws to the obtained reluctance network leads to an algebraic system of equations. The resolution of this system gives the nodal values of the magnetic potential from which we can derive the flux density distribution and the fluxes.

\section{A. 3D meshing of the studied inductor}

To mesh the complex geometry of the studied inductor, we perform a cut in cylindrical coordinates (longitudinal $z$, radial $r$ and ortho-radial $\theta$ ). Hence, the volume element has the form of a hollow cylinder sector shown in Fig.3, where:

$\alpha_{e}$ : Opening angle;

$r_{\text {out }, e}$ : Outer radius of element;

$r_{\text {int }, e}$ : Inner radius of element;

$l$ : axial length of element. 


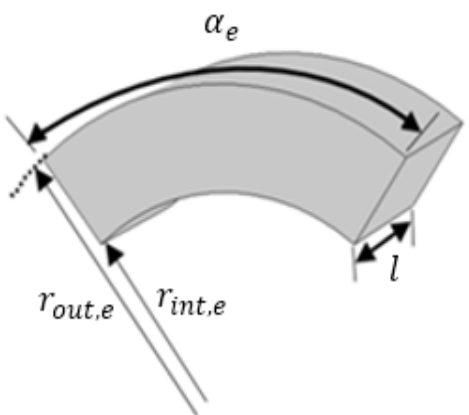

Fig.3. Hollow cylinder sector element

The inclined SC bulk is by far the most complicated region to mesh, so we need some geometry simplification as shown in Fig.4. This mesh of the SC bulk looks like a staircase.

On Figs. 5 and 6 respectively we show a cut example within the planes $(r, z)$ and $(r, \theta)$. Along the longitudinal axis $(z)$ the order of appearance of superconducting elements of the screen is highlighted on Fig.6. (a, b, c, d, e, f).

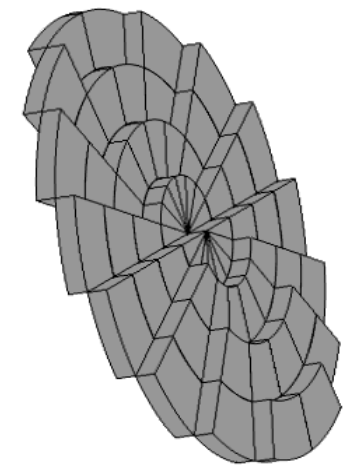

Fig.4.The approximate 3D form of the SC bulk

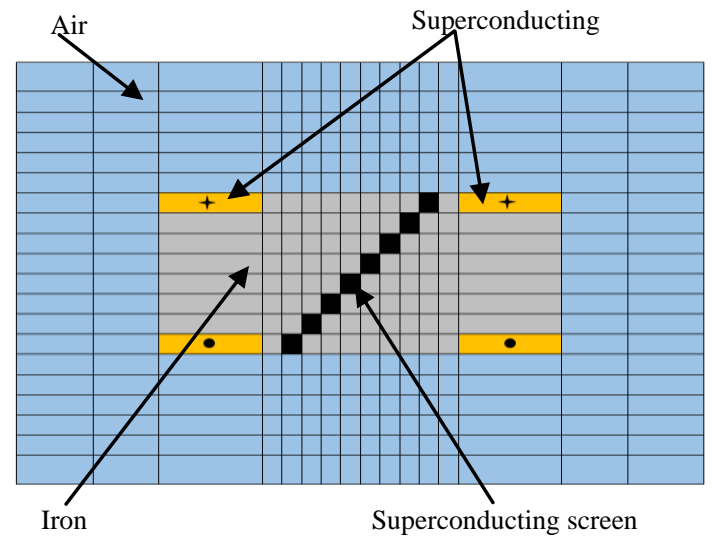

Fig.5. Inductor mesh in the plane $(r, \mathrm{z})$.



(a)

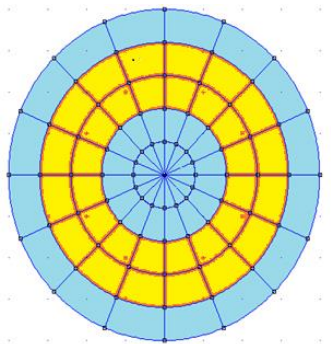

(b)

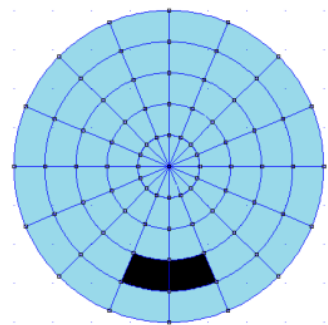

(c)

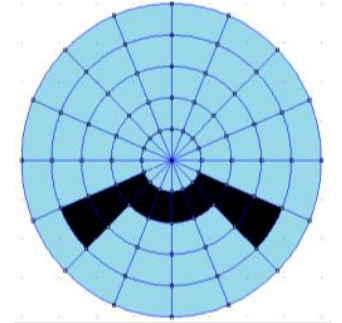

(e)

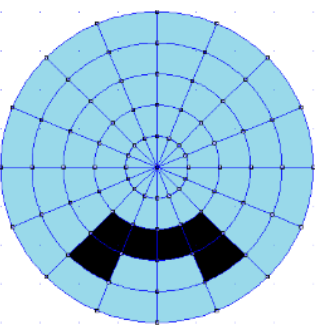

(d)

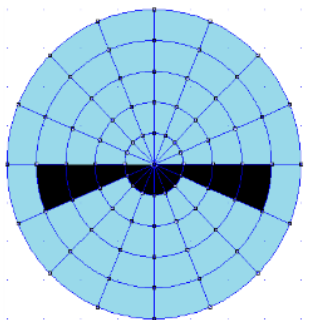

(f)
Fig.6.Inductor mesh in the plane $(r, \theta)$

To better represent the SC screen, the number of elements in the longitudinal direction between the coils is high compared to the other parts of the inductor.

\section{B. $3 D$ model of element}

To calculate the three components of the magnetic field in the volume element mesh of Fig.3, one must know all the magnetic and geometric properties of the elements. This element is modeled by six reluctances (two per direction) leading to six peripheral nodes connected to the adjacent elements. All reluctances are connected to the central node (material node) representing this element, Fig.7. In addition, the source of the magnetic field is represented by a m.m.f in series with reluctances in the appropriate directions. An element without $\mathrm{mmf}$ is said "passive", otherwise it is said "active".

The reluctances $R_{r}, R_{\theta}, R_{Z}$ in radial, ortho-radial and longitudinal directions respectively are calculated by [8]:

$$
R_{e r}=\frac{1}{\alpha_{e} \mu_{e} l} \ln \frac{r_{\text {out }, e}}{r_{\text {int }, e}}
$$

in the radial direction $(r)$,

$$
R_{e \theta}=\frac{\alpha_{e}}{\mu_{e} l} \frac{1}{\ln \frac{r_{o u t, e}}{r_{\text {int }, e}}}
$$

in the ortho-radial direction $(\theta)$ and

$$
R_{e z}=\frac{2 l}{\mu_{e} \alpha_{e}\left(\left(r_{\text {out }, e}\right)^{2}-\left(r_{\text {int }, e}\right)^{2}\right)}
$$

in the longitudinal direction $(z)$, where $\mu_{e}$ is the permeability of element. 


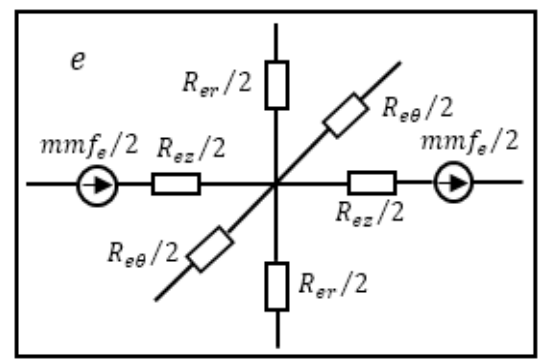

Fig.7. 3D model of active element (with $\mathrm{mmf}$ )

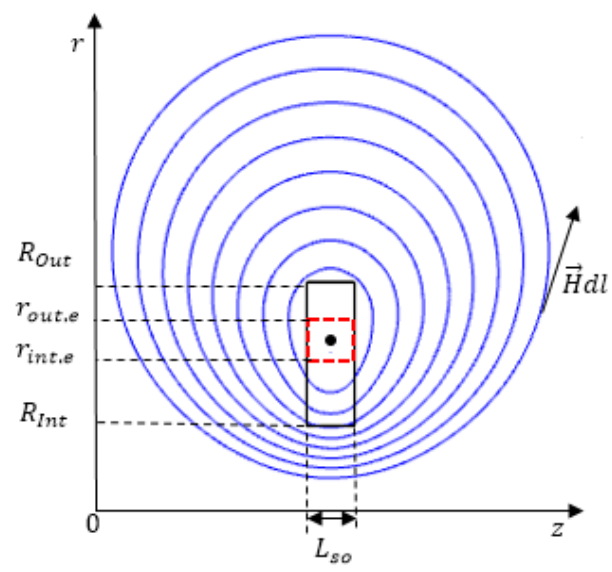

Fig.8. Field lines produced by a coil in an axisymmetric plane.

\section{Modeling of field sources}

In addition to their reluctances, two mmfs are added in the longitudinal direction for the source elements located in the coils region. According to the of Ampere's law, the circulation of the magnetic field along a closed contour equals the current surrounded by this contour line, Fig. 8 .

$$
\oint \vec{H} \cdot \overrightarrow{d l}=I
$$

The coil is subjected to a current density $J_{\theta}$ such as:

$$
\begin{gathered}
\vec{J}=J_{\theta} \overrightarrow{e_{\theta}} \\
I=\iint J_{\theta} d r d z
\end{gathered}
$$

The mmF in a coil's element $e$ is:

$$
\begin{aligned}
& F_{e}=\int_{r}^{R_{\text {Out }}} J_{\theta} L_{\text {so }} d r=-r J_{\theta} L_{\text {so }}+J_{\theta} L_{\text {so }} R_{\text {Out }} \\
& \text { If } \quad r<R_{\text {Int }}, \text { (7) becomes: } \\
& F_{e}=\int_{R_{\text {Int }}}^{R_{\text {Out }}} J_{\theta} L_{\text {so }} d r=J_{\theta} L_{\text {so }}\left(R_{\text {Out }}-R_{\text {Int }}\right)=F_{\text {emax }}
\end{aligned}
$$

Where $L_{s o}$ is the length of the coil.

Using (7) and (8), the expression of the MMF in the center of the mesh element in the coil region is then:

$$
F_{e}=\left\{\begin{array}{c}
F_{\text {emax }}, \text { if } r<R_{\text {Int }} \\
F_{\text {emax }}\left(1-\left(\frac{\frac{\left(r_{\text {out }, e}-r_{\text {int }, e}\right)}{2}+\left(r_{\text {int }, e}-R_{\text {Int }}\right)}{\left(R_{\text {Out }}-R_{\text {Int }}\right)}\right)\right)
\end{array}\right.
$$

where $R_{\text {Out }}$ and $R_{\text {Int }}$ are respectively the outer and inner radius of the coil.

\section{Equations of $3 D$ element}

In the element reluctance network of Fig. 7, the magnetic potential at the center of the element (material node) is noted $u_{e}$ and those at the connection nodes with the adjacent elements are noted $\left(u_{1 r}, u_{2 r}\right),\left(u_{1 \theta}, u_{2 \theta}\right)$ and $\left(u_{1 z}, u_{2 z}\right)$. These potentials are directly lied to the branches defined by the reluctances $\left(R_{1 r}, R_{2 r}\right),\left(R_{1 \theta}, R_{2 \theta}\right)$ and $\left(R_{1 z}, R_{2 z}\right)$. In a "passive" element, the relation between the magnetic potentials and the magnetic fluxes $\left(\varphi_{1 r}, \varphi_{2 r}\right),\left(\varphi_{1 \theta}, \varphi_{2 \theta}\right)$ and $\left(\varphi_{1 z}, \varphi_{2 z}\right)$, can be written as:

$$
\left(\left(R_{i r}+R_{e r}\right) / 2\right) \varphi_{i r}=u_{e}-u_{i r} \quad i=1,2
$$

along the radial direction,

$$
\left(\left(R_{i \theta}+R_{e \theta}\right) / 2\right) \varphi_{i \theta}=u_{e}-u_{i \theta} \quad i=1,2
$$

along the ortho-radial direction and

$$
\left(\left(R_{i z}+R_{e z}\right) / 2\right) \varphi_{i z}=u_{e}-u_{i z} \quad i=1,2
$$

along the longitudinal direction.

For an "active" element having mmfs given by (9), we can write:

$\left(\left(R_{i z}+R_{e z}\right) / 2\right) \varphi_{i z}=u_{e}-u_{i z}-\left|m m f_{e} / 2\right| \quad i=1,2$

Moreover, the magnetic flux conservation in the element leads to:

$$
\sum_{i=1}^{2} \varphi_{i r}+\varphi_{i \theta}+\varphi_{i z}=0
$$

\section{E. Global system of equations}

Rewriting equations (10)-(13) allows the determination of the magnetic fluxes $\left(\varphi_{1 r}, \varphi_{2 r}\right),\left(\varphi_{1 \theta}, \varphi_{2 \theta}\right)$ and $\left(\varphi_{1 z}, \varphi_{2 z}\right)$ expressions as a function of the permeances (inverse of the reluctances) and the mmfs. Replacing these expressions in (14) leads to a relation between the mmfs ( $F_{e}$ for active element and 0 for passive one) and the magnetic potential. Furthermore, 6 extra equations are determined using the continuity of the flux at a connection node of 2 adjacent elements. From the former relations, one can establish an algebraic system of equations whose unknowns are the magnetic potentials at the nodes of the mesh. This system is written in matrix form as:

$$
[\mathrm{P}]\{U\}=\left\{\mathrm{F}_{S}\right\}
$$


Where: $[\mathrm{P}],\{U\}$ and $\left\{\mathrm{F}_{s}\right\}$, are respectively the permeance matrix, the vector of nodal values of the magnetic potential and the vector of flux sources.

The matrix $[\mathrm{P}]$ is constant as we consider a linear case in this study. For the resolution of (15), a null potential is imposed in a reference node.

\section{ELECTROMAGNETIC QUANTITIES}

The resolution of (15) gives the distribution of the magnetic potential in all material nodes of the 3D mesh. The electromagnetic characteristics of the studied machine are then computed with these nodal values.

\section{A. Magnetic flux density components}

For an elements $(e)$ having a cross section $S_{e}$ along a given direction (radial, orthoradial or longitudinal), the flux density is determined using the flux through the branch which connects element $(e)$ to its adjacent element $(k)$ in the considered direction. It is computed by:

$$
B_{e(r, \theta, z)}=\frac{\varphi_{e k}}{S_{e}}
$$

Where $\varphi_{e k}$ is the flux through the branches connecting elements $(e)$ and $(k)$ :

$$
\varphi_{e k}=\frac{\left(U_{k}-U_{e}\right)}{R_{e k}}
$$

$R_{e k}$ is the equivalent reluctance of the considered branch.

\section{B. Induced emf}

The induced emf in phase $i$ of the armature winding $e_{i}(t)$ is expressed by:

$$
e_{i}(t)=-\frac{d \emptyset_{i}(t)}{d t}
$$

$\emptyset_{i}$ is the flux linkage through phase $i$. It is calculated by integrating the radial flux density over a pole surface located at the bore radius $R_{a}$ of the armature. If we consider a fullpitch coil of $N_{s}$ turns, the flux $\emptyset_{s k}$ embrassed by this coil is given by:

$$
\emptyset_{s k}(t)=N_{s} \int_{-L / 2}^{L / 2} \int_{0}^{\pi} B_{r}\left(R_{a}, \theta+\Omega t, z\right) R_{a} d \theta d z
$$

Then the flux per phase is:

$$
\emptyset_{i}(t)=\sum_{k=1}^{q} \emptyset_{s k}(t)
$$

$q:$ number of coils in series per phase;

$L$ : active length of armature;

$\Omega$ : angular velocity of the rotor

\section{RESULTS AND VALIDATION}

The results obtained using the reluctance network are compared to those issued from 3D finite element computations and to experiments. Two cases are considered: the first one concerns the study of the inductor alone (stator armature removed) and the second the full machine under no-load condition.

\section{A. Finite element analysis}

The studied inductor has been modelled by finite elements using COMSOL ${ }^{\circledR}$ Multiphysics software. In this model, we have considered:

- the two SC solenoids are supplied by uniform current density across their cross section area;

- the screening property of the SC bulk is accounted for by considering a low relative permeability $\mu_{r}=10^{-3}$.

- a smooth ferromagnetic yoke is considered for the armature so slotting effects are neglected.

Hence, the problem we have solved by the $3 \mathrm{D}$ FE method is linear magnetostatic with no armature currents.

Figure 9 presents the FE mesh used for the two study cases. To get reliable results, the mesh is refined until convergent results are obtained.

The number of elements in the inductor alone and the inductor with the smooth iron armature are (132696 elements) and (336795 elements) respectively. The computation time is about $113 \mathrm{~s}$ for the inductor alone and $312 \mathrm{~s}$ when the armature is added. For the same study cases, the computation time obtained with the developed reluctance network is about $21 \mathrm{~s}$ (19488 nodes for both inductor alone and full machine). These computations are performed using a 8 GB PC, Intel CORE i3 processor.



(a)

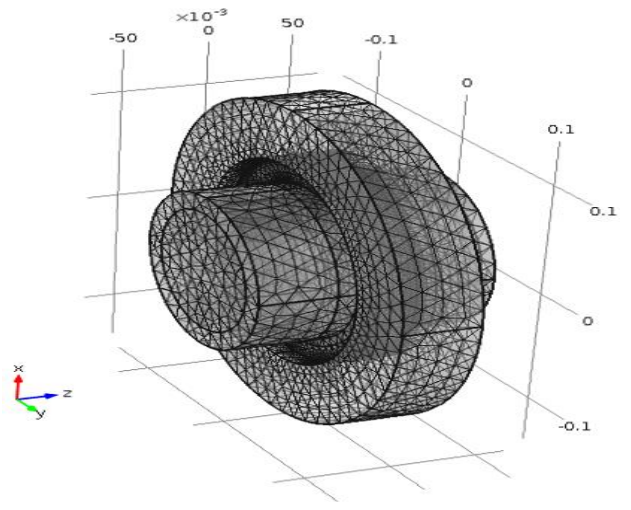

(b)

Fig. 9. 3D mesh of the studied device: a) inductor alone; b) with smooth iron armature. 


\section{B. Study of the inductor alone}

In order to check its good operation (so the shielding properties of the SC bulk), the inductor has been tested alone (without external armature). Figure 10 shows the test bench where one can see the constructed inductor which has been inserted in a cryostat of $15 \mathrm{~mm}$ wall thickness.

The tests of the inductor alone have been performed at $77 \mathrm{~K}$; the inductor being cooled in liquid Nitrogen. At $77 \mathrm{~K}$, the $\mathrm{NbTi}$ coils are not superconducting (their operating temperature is $4.2 \mathrm{~K}$ ) but the YBCO bulks are in superconducting state. The tests have been carried out with a current $I e=4 \mathrm{~A}$ which corresponds to a current density equals to $10.4 \mathrm{~A} / \mathrm{mm}^{2}$ in the $\mathrm{NbTi}$ conductor which results in a low flux density. Hence, the iron parts of the inductor have a high relative permeability taken equal to 1000 in the FE and the RN models.

A Hall probe has been placed on the cryostat surface at a radius of $83 \mathrm{~mm}$ to measure the radial flux density. It has been moved in the axial and ortho-radial directions using a 3-axis (xyz) table.

Figure 11 presents the radial flux density vs. angular position waveform along a circle located in the middle of the inductor (at $z=0$ ). The results show a good agreement between the computed and the measured curves. It can be seen that the inductor effectively creates 2-poles and the radial flux density shape is almost rectangular.

Figure 12 presents the radial flux density variation along a line representing the active axial length. Again, a good agreement is observed between the computed and the measured curves.

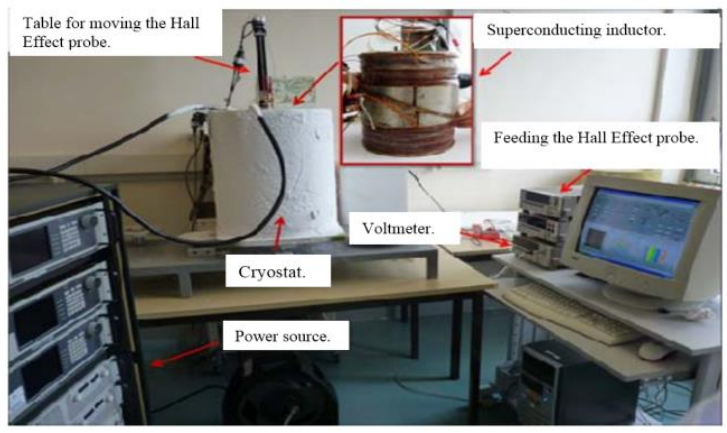

Fig.10. Photograph of the test bench [5]

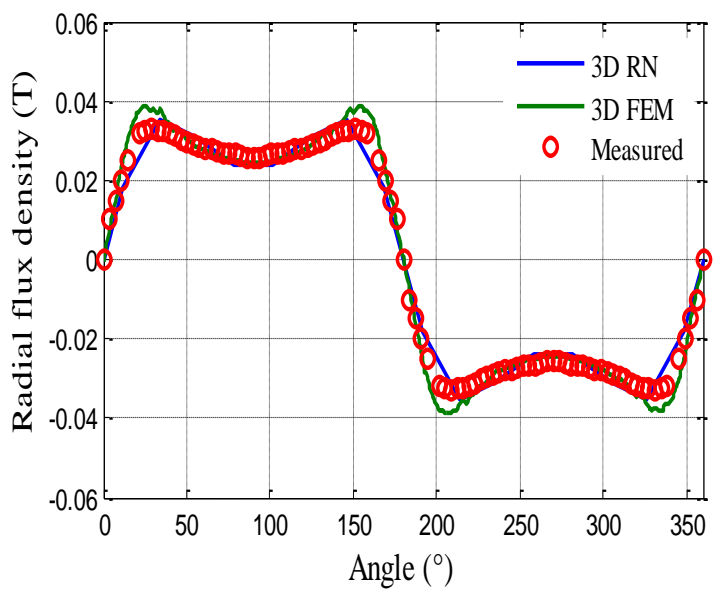

Fig.11. Radial flux density component on half of pole at $z=0$.

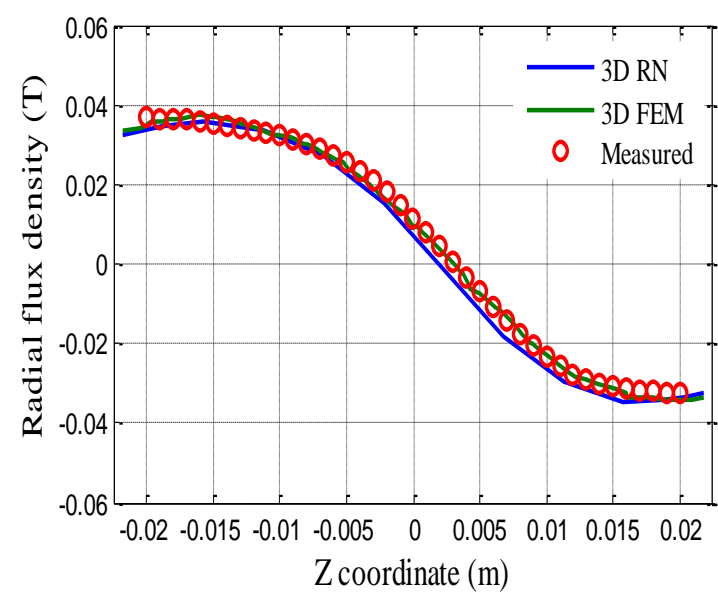

Fig.12. Radial flux density component as function of $z$ at $\theta=0$.

\section{Study of the machine at no-load}

The machine is now fully assembled with the inductor cooled in liquid helium at $4.2 \mathrm{~K}$. The armature of the machine consists of 48 semi-closed slots where the 2-pole, 3-phase winding is placed. A single-layer, full-pitch configuration has been adopted which results in 8 coils in series per phase. Each coil has $N_{s}=105$ turns in series, so the total number of turns in series per phase is 840 .

In the RN and FE models, a smooth armature is considered so the slotting effect is neglected. Hence, the radial flux density, flux and no-load phase emf of the machine are obtained using relations (16)-(20).

The current in the $\mathrm{SC}$ coils is set to $I e=97 \mathrm{~A}$ which results in a current density of $250 \mathrm{~A} / \mathrm{mm}^{2}$ in the NbTi conductor. The iron parts of the inductor are saturated but these of the armature are not. Hence, in both linear RN and FE models, the relative permeability is set to $\mu_{r}=4$ in the inductor iron and $\mu_{r}=1000$ in the iron armature.

In Figure 13, we compare the RN and FE computations of the radial flux density vs. angular position waveform for $z=0$ (at the middle of the inductor). A good agreement is noticed between the RN and the FE computations. The peak value of the radial flux density is almost equal to $0.8 \mathrm{~T}$ at $r=75 \mathrm{~mm}$ and $0.6 \mathrm{~T}$ at $r=87 \mathrm{~mm}$. This shows the influence of the cryostat in SC machines, which makes them suitable for large power applications for which the airgap is anyhow large.

In Figure 14, we compare the RN and FE computations of the radial flux density vs. angular position waveform for $z= \pm L_{A} / 2$ (at the ends of the inductor). In contrast to Figure 13 where the computation is done at $z=0$, the radial flux density waveforms given in Figs 14.a and b are not symmetrical and present a mean value (positive at $z=L_{A} / 2$ and negative at $z=-L_{A} / 2$ ).

The total flux under one pole computed for several positions of the inductor is symmetrical as shown in Figure 15. Hence, the studied machine behaves like a standard radial flux synchronous machine. 


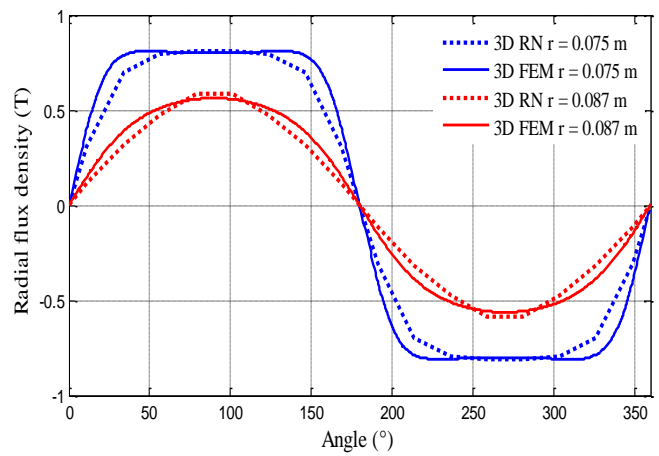

Fig.13. Radial flux density vs. angle at $z=0(I e=97 \mathrm{~A})$

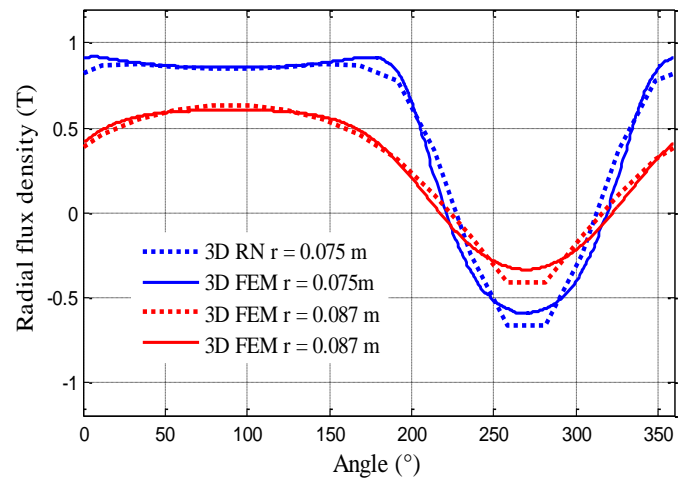

(a)

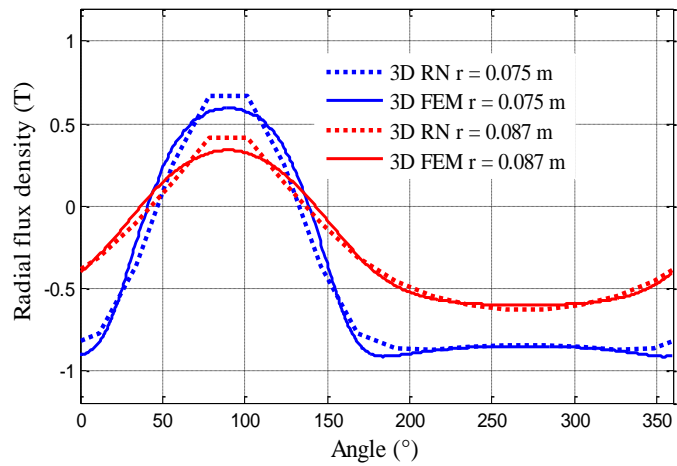

(b)

Fig.14. Radial flux density vs. angle $(I e=97 \mathrm{~A})$ a) $z=L_{A} / 2$ b) $z=-L_{A} / 2$

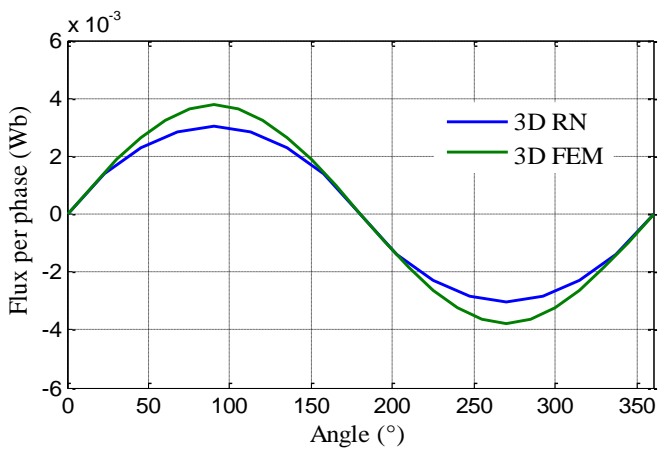

Fig.15. Flux under one pole of the machine vs. rotor angular position.

The machine has been tested under no-load conditions for a rotating speed of $500 \mathrm{rpm}$. The emf has been computed and measured for an inductor current $I e=97$
Figure 16 presents the computed and measured emfs waveforms. It can be seen that the RN and FE computations are consistent with the measurements. Because of the large magnetic air-gap, the emfs are almost sinusoidal although the saturation level is very high. In Figure 17 we compare the emfs harmonics spectra obtained using the linear RN model and the FEM in saturated case. It can be seen that taking a relative permeability $\mu_{r}=4$ in the inductor iron is enough to get reliable results.

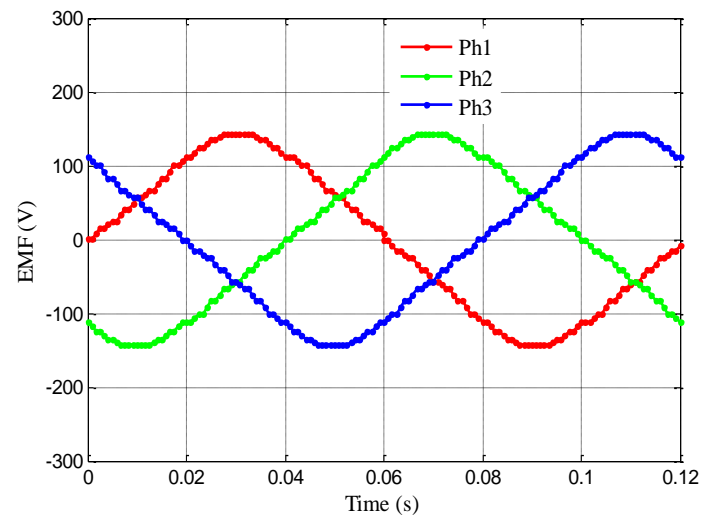

(a)



(b)

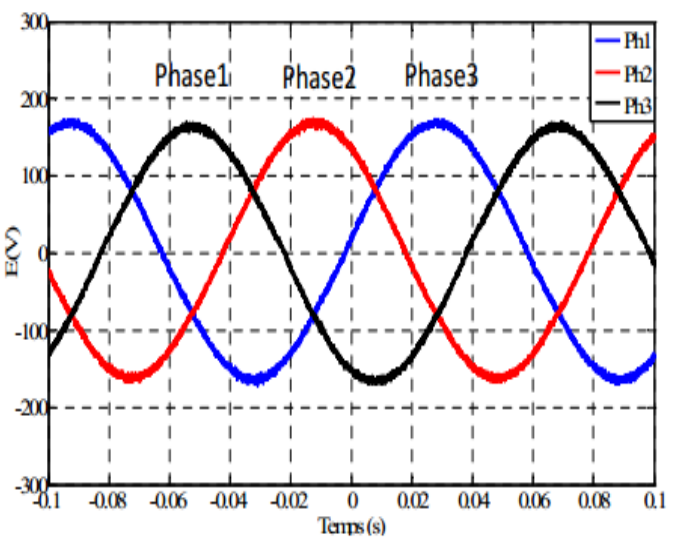

(c)

Fig. 16 No-load emf at $500 \mathrm{rpm}$ and $I e=97 \mathrm{~A}$ [5] a) Computed with the $\mathrm{RN}$ model

b) Computed with the FE model with $\mathrm{B}(\mathrm{H})$ curve c) Measured 


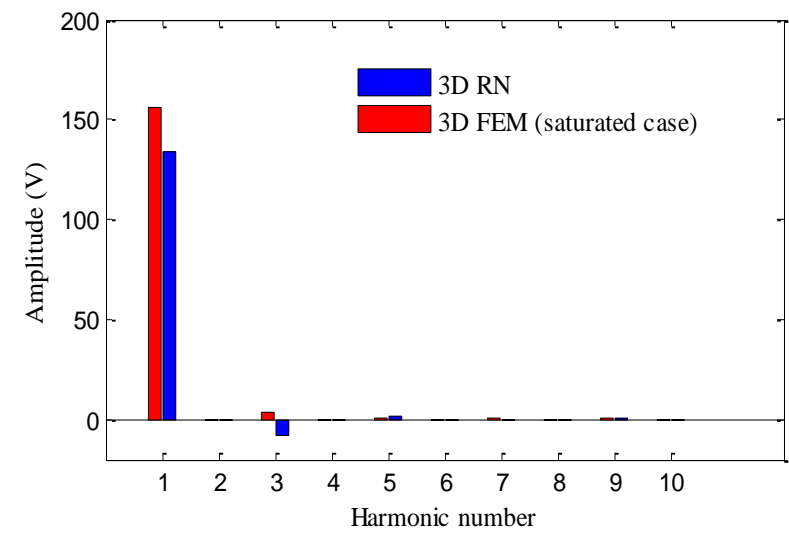

Fig .17. Harmonics spectras of emf. Blue: RN . Red: FEM (saturated case)

\section{STUdy OF THE SHIELDING PROPERTIES OF THE SC BULK}

In the RN and FE models of the inductor presented above, the shielding properties of the SC bulk have been considered using a low relative permeability. This approach is well suited for quickly sizing the studied machine using magnetostatics. In reality, the study of the electromagnetic behavior of a SC material is much more complicated. Indeed, it is described by a highly non-linear diffusion equation due to the non-linear $\mathrm{E}(\mathrm{J})$ curve of the SC material [16], [17], [18].

We propose in this section to study the magnetic field diffusion in the SC bulk of our inductor submitted to an external variable magnetic field. The aim here is to show the limits of the shielding properties of the SC bulk according to the applied external field. Knowing these limits allow the designer of the studied machine to set the maximum allowable current in the solenoids of the inductor.

\section{A. Magnetic field diffusion in the SC bulk}

We propose to deal with the problem shown on Fig.17. The $\mathrm{SC}$ bulk is subjected to an external magnetic field in the $\mathrm{z}$ direction (as created by the solenoids of the actual inductor). The bulk is surrounded by a cylinder on which we set the boundary conditions. The external time-varying flux density is a ramp:

$$
B_{a p p}(t)=a * t
$$

where $a$ is the ramp rate in $\mathrm{T} / \mathrm{s}$.

This is actually the situation of the SC bulk in our inductor since the solenoids are energized using a dc supply delivering a current ramp until the desired current value is reached.

The diffusion of the electromagnetic field is computed by finite elements using a H-formulations in which the magnetic field $\vec{H}$ is the state variable [18]. This formulation seems to give consistent results and has good convergence properties. Combining Ampere's and Faraday's laws, the governing PDE for the magnetic field diffusion is:

$$
\operatorname{curl}(\rho(|J|) \operatorname{curl}(\vec{H}))=-\mu_{0} \frac{d \vec{H}}{d t}
$$

where $\rho(|J|)$ represents the nonlinear resistivity of the SC bulk.

Indeed, the electric field vs. current density constitutive law in a superconductor can be written as

$$
\vec{E}=\rho(|J|) \vec{J}
$$

The power law is usually employed to describe the electric behavior of a high temperature superconductor, which leads to:

$$
\rho(|J|)=\frac{E c}{J c(B)}\left(\frac{|J|}{J c(B)}\right)^{(n-1)}
$$

In (24), $E c=10^{-4} \mathrm{~V} / \mathrm{m}$ is the critical electric field and $J c$ the critical current density which depends on the magnetic flux density norm $B$. The index of the power law is set to $n=30$ in this study.

Fig. 18 shows the $\mathrm{Jc}(\mathrm{B})$ curve of a YBCO bulk material cooled in liquid helium at $4.2 \mathrm{~K}$, [13]. The critical current density under self-field condition is about $5.10^{4} \mathrm{~A} / \mathrm{mm}^{2}$, this value decreases with the increase of the applied flux density.

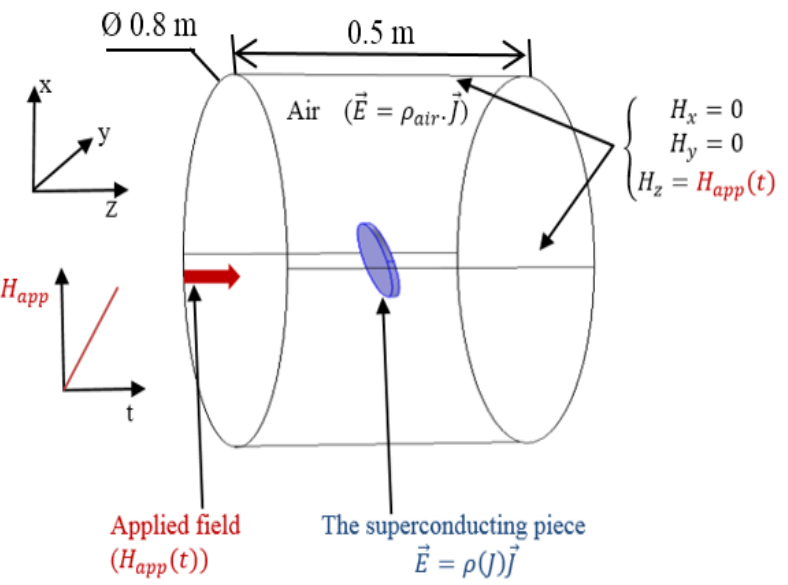

Fig. 17 Cartesian 3D problem: SC bulk subjected to a field ramp in the $\mathrm{z}$ direction.

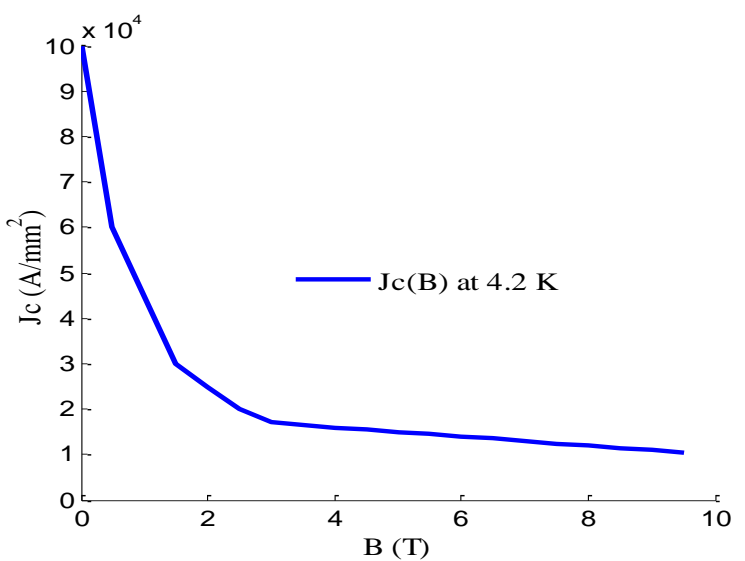

Fig. 18. Jc(B) curve of bulk YBCO at 4.2K [13]. 
In the FE model implemented under COMSOL software environment, this $\mathrm{Jc}(\mathrm{B})$ curve has been tabulated so a linear interpolation between 2 successive values is used in the iterative solution procedure of the non-linear 3D FE problem.

\section{B. Results}

As stated above, the aim here is to check the shielding properties of the YBCO bulk to confirm the good operation of the studied inductor. Hence the magnetic field distribution inside the SC bulk is the representative quantity to evaluate the quality of the shielding.

The ramp rate is low and fixed to $a=0.2 \mathrm{~T} / \mathrm{s}$.

Fig. 19 shows the distribution of the flux density normal to the plane in the middle of the YBCO bulk for an applied flux density $\mathrm{B}_{\text {app }}=3 \mathrm{~T}$ (at $\left.\mathrm{t}=15 \mathrm{~s}\right)$.

The penetration depth of the magnetic field toward the center of the bulk is very low $(<8 \mathrm{~mm})$ which shows that the SC bulk well fulfills its shielding function. This can also be seen from the curves of Fig.20 where the normal flux density is plotted at different instants on a line situated in the middle of the YBCO bulk. As expected, the curves look like the ones we obtain using the critical state model.

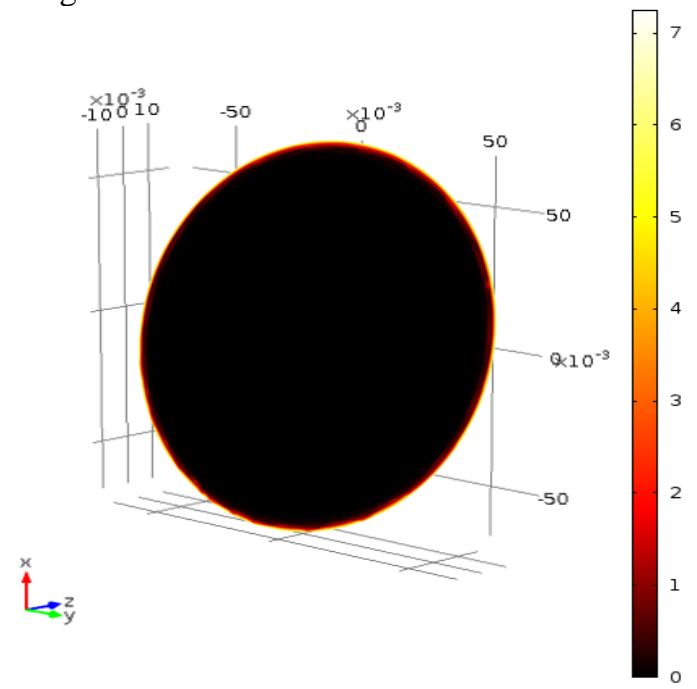

Fig. 19 Distribution of the normal component of B in the middle plane of the YBCO bulk at $\mathrm{t}=15 \mathrm{~s}$ and $B_{a p p}=3 T$

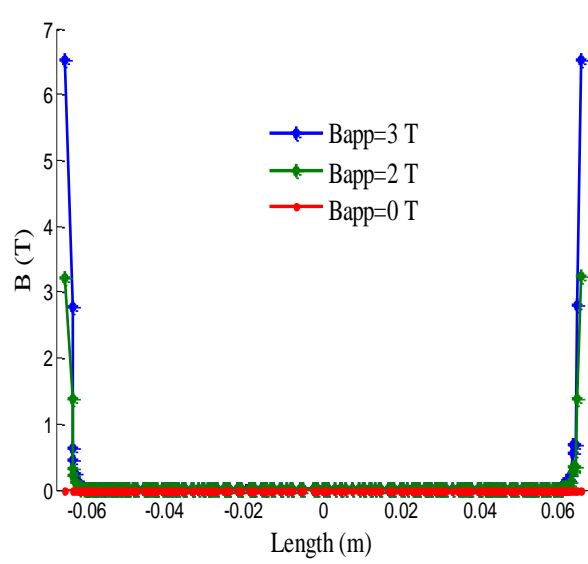

(a)

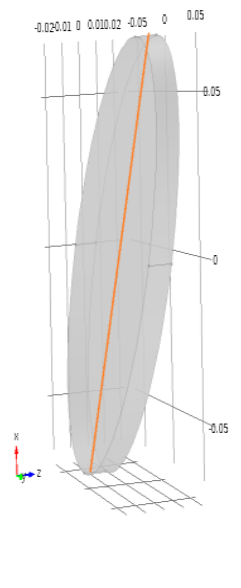

(b)
Fig. 20. (a) Normal flux density as function of $B_{a p p}$, (b) Computation line inside the YBCO bulk.

\section{CONCLUSION}

A new superconducting inductor is modeled by 3Dreluctance network. The results obtained from the developed model are validated through FE computations and experiments.

The developed model can be easily used to optimize this superconducting inductor because the calculation time is low compared to the 3D finite elements.

To confirm that the YBCO bulk fulfills its shielding function at 3T, we have developed a 3D FE model to compute the diffusion of the magnetic field in the bulk. This model takes into account the actual $\mathrm{E}(\mathrm{J})$ power law of $\mathrm{YBCO}$ and the dependence of the critical current density vs. the flux density. In future works, the whole machine under load conditions will be investigated by a non-linear reluctance network to consider the saturation of the iron parts.

\section{REFERENCES}

[1] E. Ailam, D. Netter, J. Leveque, B. Douine, P. J. Masson and A. Rezzoug, "Design and Testing of a Superconducting Rotating Machine," IEEE Transactions on Applied Superconductivity, vol. 17, no. 1, pp. 2733, March 2007.

[2] R. Qu, Y. Liu and J. Wang, "Review of Superconducting Generator Topologies for Direct-Drive Wind Turbines," IEEE Transactions on Applied Superconductivity, vol. 23, no. 3, pp. 5201108-5201108, June 2013.

[3] P. Masson, J. Leveque, D. Netter, and A. Rezzoug, "Experimental study of a new kind of superconducting inductor", IEEE Trans. Applied Superconductivity, vol. 13, no. 2, pp. 2239-2242, Jun. 2003.

[4] P. Tixador, "Superconducting electrical motors", International Journal of Refrigeration, CNRS-CRTBT/LEG, B.P. 166, 38042 Grenoble Cedex 09, France, 22 (1999) 150-157.

[5] R. Alhasan, T. Lubin, B. Douine, Z. M. Adilov and J. Lévêque, "Test of an Original Superconducting Synchronous Machine Based on Magnetic Shielding," IEEE Transactions on Applied Superconductivity, vol. 26, no. 4, pp. 1-5, June 2016.

[6] R. Alhasan, T. Lubin and J. Leveque, "Study and test of a new superconducting inductor structure for a synchronous machine," Electrical Sciences and Technologies in Maghreb (CISTEM), 2014 International Conference on. IEEE, Tunis, 2014, pp. 1-7.

[7] P. J. Masson and C. A. Luongo, "High power density superconducting motor for all-electric aircraft propulsion", IEEE Transactions on Applied Superconductivity, vol. 15, no. 2, pp. 2226-2229, June 2005.

[8] V. Ostovic, "Dynamics of Saturated Electric Machines," NewYork, Springer-Verlag, 1989

[9] B. Nedjar, S. Hlioui, L. Vido, Y. Amara, and M. Gabsi, "Hybrid Excitation Synchronous Machine Modeling Using Magnetic Equivalent Circuits," International Conference on Electrical Machines and Systems (ICEMS), Sept 2011, Beijing, China.

[10] K. Hoang, L. Vido, M. Gabsi and F. Gillon, "3D modeling of double excitation synchronous motor with reluctance network,"International Conference on Electrical Machines (ICEM), Berlin, Sept 2014, pp. 25982604.

[11]H. W. Derbas, J. M. Williams, A. C. Koenig and S. D. Pekarek, "A Comparison of Nodal- and Mesh-Based Magnetic Equivalent Circuit Models ", IEEE Transactions on energy Conversion, Vol. 24, No. 2, pp.388-396, June 2009.

[12] Michelle L. Bash, Joshua M. Williams and Steven D. Pekarek, "Incorporating Motion in Mesh-Based Magnetic Equivalent Circuits," IEEE Transactions on energy Conversion, Vol. 25, No. 2, pp.329-338, June 2010.

[13] M., Teshima Morita, "Development of oxide superconductors - High- Tc bulk superconductor, QMG, and its magnetic applications - High-Tc b," 2006. Available: http://en.zl50.com/2012031912347806.html. [Accessed: 23-mai-2012].

[14] W. Ta and Y. Gao, "Electromagnetic-Mechanical Coupling Analysis of $\mathrm{Nb} 3 \mathrm{Sn}$ Superconducting Strand," IEEE Transactions on Applied Superconductivity, vol. 26, no. 4, pp. 1-5, June 2016. 
[15] Z. Hong, Z. Jin, M. Ainslie, J. Sheng, W. Yuan and T. A. Coombs, "Numerical Analysis of the Current and Voltage Sharing Issues for Resistive Fault Current Limiter Using YBCO Coated Conductors," in IEEE Transactions on Applied Superconductivity, vol. 21, no. 3, pp. 1198-1201, June 2011.

[16] G. Iannone, S. Farinon, G. De Marzi, P. Fabbricatore and U. Gambardella, "Modeling Experimental Magnetization Cycles of Thin Superconducting Strips by Finite-Element Simulations," in IEEE Transactions on Applied Superconductivity, vol. 25, no. 1, pp. 1-7, Feb. 2015.

[17] J. López, R. Maynou, X. Granados, R. Bosch and J. Grau, "Finite Element Analysis of Thermal and Electromagnetic Relaxation Processes in a YBCO Superconducting Pellet. Trapped Fields and Currents Distributions Differences," IEEE Transactions on Applied Superconductivity, vol. 23, no. 3, pp. 4901104-4901104, June 2013.

[18] R. Brambilla, F. Grilli, and L. Martini, "Development of an edgeelement model for AC losses computation of high-temperature superconductors," Supercond. Sci. Technol., vol. 19, pp. 1-9, 2006. 